Behavioural symptoms among people with severe and profound intellectual disabilities: a 26-year

\title{
follow-up study
}

\author{
CHRISTINA L. THOMPSON and ANDREW REID
}

\begin{abstract}
Background Verylittle is known about the natural history of challenging behaviour and psychiatric disorder in people with severe and profound degrees of intellectual disability.
\end{abstract}

Aims To clarify the natural history of challenging behaviour and psychiatric disorder in this population through a longterm prospective cohort study over a 26-year period.

Method One hundred individuals with severe or profound intellectual disability were randomly selected in 1975. Their behaviour was recorded through carer and psychiatrist ratings using the Modified Manifest Abnormality Scale of the Clinical Interview Schedule. The presence and severity of psychiatric disorder were also recorded. The study was repeated in 1981/82 and 1992/93. We repeated the study again in 200I, supplementing the original observational data with the Checklist of Challenging Behaviour.

Results Behavioural symptomatology is remarkably persistent, particularly stereotypy, emotional abnormalities, eye avoidance and overactivity, although the severity of overall psychiatric disorder does show some abatement through time.

Conclusions These findings influence the prospects of success in relocating adults with severe and profound degrees of intellectual disability back into the community.

Declaration of interest None.
Psychiatric symptoms and challenging behaviour often coexist in adults with severe and profound intellectual disabilities. These phenomena tend to persist over time (Kiernan \& Alborz, 1996; Nottestad et al, 2000) and can be difficult to distinguish (Moss et al, 2000). It is important to establish the natural history of these behavioural symptoms and psychiatric disorders so that we can assess the efficacy of management and treatment procedures. This is particularly relevant because people with severe intellectual disability are now living longer (Day \& Jancar, 1994) and the needs of this ageing population are becoming increasingly important (Holland, 2000). However, it is an underresearched area and this cohort study, which charts the challenging behaviour and psychiatric symptoms of a group of adults with severe and profound intellectual disabilities over a 26-year period, offers a unique perspective on these issues.

\section{METHOD}

In $1975 / 76$ the second author, with colleagues, randomly selected 100 adults with severe and profound degrees of intellectual disabilities and studied their behavioural patterns and psychiatric syndromes (Reid et al, 1978).

The data collection was repeated in 1981/82 (Reid et al, 1984) and again in 1992/93 (Reid \& Ballinger, 1995). We have repeated the assessments once again and present the results of a comparison of the $1975 / 76$ data with the 2001 data to establish possible changes in behavioural and psychiatric patterns.

\section{Design and participants}

The study is a longitudinal, withinparticipant design, following the cohort over a 26-year period. The original 1975/ 76 sample consisted of 100 individuals living at a long-stay hospital for people with intellectual disabilities: 45 males and 55 females with a mean age of 35 years (range 17-78), 49 of them with severe intellectual disability and 51 with profound intellectual disability. Subsequently, two individuals were excluded because it emerged that they were functioning above the 'severe' level of intellectual disability. Of the remaining 98, 54 are still living. One individual was not followed up in 2001 because she was relocated repeatedly throughout the data collection period. The study is, therefore, a behavioural comparison of the 53 survivors in 2001 from the original cohort with those same 53 individuals in 1975.

The present sample consisted of 21 males and 32 females with a mean age of 57.6 years (range 42-92): 26 (49.1\%) with severe intellectual disability, 18 (34\%) with profound intellectual disability and 9 $(\mathbf{1 7 \%})$ whose level of intellectual disability varied on the border between severe and profound. In 28 out of $53(52.8 \%)$ the intellectual disability was of unknown aetiology, with other causes including hydrocephalus (1 out of $53,1.9 \%$ ) and Down's syndrome (4 out of $53,7.5 \%$ ).

Forty-two of the cohort are currently living in the community, with the remainder either living in the same long-stay hospital as in $1975 / 76$ or in specialist accommodation. As a group, the participants have been resident in hospital for a mean of 36.7 years (range 10-67).

\section{Measures taken}

In both the 1975/76 and the 2001 study, the following demographic data were noted: age, gender, cause of intellectual disability (if known), level of intellectual disability, continence level, whether the individual had epilepsy and whether they had significant impairment of vision, hearing or mobility. We adopted the same definition of epilepsy in 2001 as in 1975/ 76 , defining a patient as having epilepsy if he or she had suffered three or more fits over the last 2 years or was receiving any anticonvulsant medication for previous epilepsy (Gunn \& Fenton, 1969).

Participants were also assessed in the following two ways. First, staff informant interviews, originally devised by Reid et al in 1978 , asked primary carers if participants had shown significant abnormality during the preceding week in relation to social withdrawal, overactivity, stereotypies, irritability, nosiness, self-injury, 
stripping, sleep disturbance or feeding disorder: ' 0 ' indicated that the symptom was absent and ' 1 ' indicated that it was present. This information represents dichotomous data and the ratings are referred to as 'carer ratings'. Second, each participant was interviewed/observed for approximately $20 \mathrm{~min}$ using the Modified Manifest Abnormality Scale (MMAS) of the Clinical Interview Schedule (Goldberg et al, 1970).

The MMAS (see Appendix) defines and records 19 behaviours and psychiatric symptoms on a five-point rating scale: a zero rating indicates that the symptoms are absent, whereas ratings of 1-4 represent increasing degrees of severity. Ballinger et al (1975) had previously shown this modified scale to be a reasonably valid and reliable instrument in this population, although the numbers involved in the original reliability study were small and correlations for some of the items, for example 'histrionic', 'anxious' and 'hostile irritability', did not reach statistical significance. Some other items, for example 'delusions, misinterpretations and thought disorder' and 'pica', were also recorded so rarely that statistical analysis made little sense. The interpretation of significant changes/lack of changes on these symptoms should therefore be viewed cautiously.

For the 1975/76 data, observations of behaviour and mental state were made by psychiatrists. For the 2001 data, these same observations were made by a research psychologist. The reliability of these ratings was not established, but the psychologist had extensive research and clinical experience with this population, with particular reference to behavioural observation. The authors, therefore, would have no concern about the psychologist's expertise in assessing and rating psychopathology. For the purposes of consistency between published papers, the psychologist's ratings are referred to in this follow-up study as 'psychiatrist ratings'.

These two sources of information were then reviewed and a decision was made by the psychiatrist/psychologist as to whether each participant showed evidence of a psychiatric disorder.

As with the previous studies, psychiatric disorder was defined as follows: 'abnormalities of emotions, behaviour, relationships, or thinking which are inconsistent with the patient's intellectual level and of sufficient duration or severity to cause persistent suffering or handicap to the person and/or distress and disturbance to those in daily contact with him/her'. This definition was derived from that of Rutter \& Graham (1968). We rated psychiatric disorder thus defined on a fivepoint scale: $0=$ no disorder, $1=$ personality quirks or eccentricities of behaviour, not amounting to overt psychiatric disorder or sufficient to cause management problems 2, 3 and 4 indicate mild, moderate and severe degrees of psychiatric disorder, respectively. The second author has used this five-point scale previously for rating psychiatric disorder in a population with intellectual disability (Ballinger \& Reid, 1977). It was used in both the $1975 / 76$ and 2001 studies to estimate, at the time of interview, the presence or absence and degree of severity of psychiatric disorder. It represents a subjective opinion by the interviewer and the data are referred to as 'overall' ratings.

In addition, for the 2001 study, data were collected using the Checklist of Challenging Behaviour (CCB; Harris et al, 1994). This checklist aims to identify: the type and number of challenging behaviours exhibited by residents and explores how often an individual displays these behaviours; if staff find these behaviours difficult to manage; and if any injuries have been caused as a result within the 3 months prior to administration of the assessment. The CCB has been found to be useful as a screening tool for both challenging behaviour and mental health problems (Jenkins et al, 1998) and it is used here to lend validity to the subjective 'overall' interviewer ratings.

The CCB is divided into two parts: Part 1 consists of 14 aggressive behaviours that involve physical contact with other people and self-injury; and Part 2 consists of 18 other types of challenging behaviours, such as absconding and stereotypical behaviours. Both parts are rated on a fivepoint scale: Part 1 for frequency, management difficulty and severity; and Part 2 for frequency and management difficulty. Within each scale, a higher number denotes a more frequent behaviour, difficult behaviour or severe injury. For example, the frequency scale ranges from ' 1 : never occurred' to '5: occurs daily or more often'.

For the purposes of this study, the rating scale was modified to ' $0-4$ ' rather than ' $1-5$ ' because it was felt that the original scale was subject to false inflation of scores. On the original scale, an individual who displayed no challenging behaviours would have received a score of 32. By modifying the scale, the same individual would now receive a more authentic score of zero; if a behaviour has not occurred, it is rated as zero across all scales.

\section{RESULTS}

\section{Other disabilities}

Of the sample, 17 (32.1\%) were epileptic, $7(13.2 \%)$ were visually impaired or registered blind, 2 (3.8\%) were hearing impaired or deaf, $36(68 \%)$ were partially or fully incontinent and $16(30.2 \%)$ had mobility difficulties or were immobile. The corresponding figures for $1975 / 76$ were $15(28.3 \%), 6(11.3 \%), 4(7.5 \%)$, $31 \quad(58.5 \%)$ and $6 \quad(11.3 \%)$. The Wilcoxon signed rank test indicated a significant increase at follow-up in the number of individuals who were immobile or who required assistance to get around. No other significant differences were found between the 1975/76 and 2001 studies for the other disabilities.

\section{Carer ratings}

Table 1 compares the carer ratings for the 53 survivors between 1975/76 (baseline) and 2001 (follow-up). Because the data for these ratings are dichotomous, correlation coefficient $\phi$ was used to explore the relationships between ratings. This indicated three categories that showed a significant correlation between assessment points: noisy, social withdrawal and overactive. It should be noted, however, that all three correlation coefficients were fairly weak. The remaining behaviours were not found to be significantly correlated despite showing similar percentages between assessment periods.

\section{Psychiatrist ratings}

Table 2 compares the interviewer ratings of manifest abnormalities between 1975/ 76 and 2001 using the Wilcoxon signed rank test. Ratings of slow, lability of mood, and depressed show a significant increase over assessment periods.

Among the other symptoms, four were not present at either period and the remainder showed no significant differences over time, indicating their persistence. Stereotypy, in particular, is present in over $60 \%$ of the cohort at both periods. 
Table I Carer ratings: baseline and follow-up comparison

\begin{tabular}{lccc}
\hline Category & $\begin{array}{c}\text { Baseline } \\
(1975) n(\%)\end{array}$ & $\begin{array}{c}\text { Follow-up } \\
(200 I) n(\%)\end{array}$ & $\begin{array}{c}\text { Correlation coefficient } \\
\phi \text { and significance }\end{array}$ \\
\hline Noisy & $19(35.8)$ & $28(52.8)$ & $0.39(P<0.005)$ \\
Social withdrawal & $10(18.9)$ & $9(17.0)$ & $0.29(P<0.05)$ \\
Overactive & $26(49.1)$ & $12(22.6)$ & $0.28(P<0.05)$ \\
Irritability & $36(67.9)$ & $25(47.2)$ & NS \\
Self-injurious behaviour & $23(43.4)$ & $10(18.9)$ & NS \\
Stereotypy & $20(37.7)$ & $22(41.5)$ & NS \\
Feeding disorder & $13(24.5)$ & $12(22.6)$ & NS \\
Sleeping disturbance & $8(15.1)$ & $8(15.1)$ & NS \\
Stripping & $6(11.3)$ & $11(20.8)$ & NS \\
\hline
\end{tabular}

Table 2 Psychiatrist ratings: baseline and follow-up comparison

\begin{tabular}{|c|c|c|c|}
\hline Category & $\begin{array}{c}\text { Baseline } \\
(1975) n(\%)\end{array}$ & $\begin{array}{l}\text { Follow-up } \\
(2001) n(\%)\end{array}$ & $\begin{array}{c}\text { Wilcoxon signed rank } \\
\text { test (two-tailed) }\end{array}$ \\
\hline Slow & $9(17.0)$ & $18(34.0)$ & $Z=-2.340(P<0.05)$ \\
\hline Lability of mood & I (I.9) & $4(7.5)$ & $Z=-2.226(P<0.05)$ \\
\hline Depressed & I (I.9) & $5(9.4)$ & $Z=-2.203(P<0.05)$ \\
\hline Stereotypy & $32(60.4)$ & $34(64.2)$ & NS \\
\hline Flattened & $15(28.3)$ & $8(15.1)$ & NS \\
\hline Eye avoidance & $14(26.4)$ & $14(26.4)$ & NS \\
\hline Overactivity & $13(24.5)$ & $7(13.2)$ & NS \\
\hline Elated & $9(17.0)$ & $6(11.3)$ & NS \\
\hline Suspicious & $8(15.1)$ & $3(5.7)$ & NS \\
\hline Distractibility & $7(13.2)$ & $3(5.7)$ & NS \\
\hline Anxious & $6(11.3)$ & $12(22.6)$ & NS \\
\hline Hostile irritability & 4 (7.5) & $4(7.5)$ & NS \\
\hline Histrionic & $3(5.7)$ & I (I.9) & NS \\
\hline Self-injurious behaviour & $3(5.7)$ & $6(11.3)$ & NS \\
\hline Pica & $2(3.8)$ & 0 & NS \\
\hline Delusions & 0 & 0 & NS \\
\hline Hallucinations & 0 & 0 & NS \\
\hline Excessive concern with body & 0 & 0 & NS \\
\hline Depressive thoughts & 0 & 0 & NS \\
\hline
\end{tabular}

Table 3 Overall psychiatric ratings: baseline and follow-up comparison

\begin{tabular}{lcc}
\hline $\begin{array}{l}\text { Overall } \\
\text { rating }\end{array}$ & $\begin{array}{c}\text { Baseline } \\
(1975) n(\%)\end{array}$ & $\begin{array}{c}\text { Follow-up } \\
(200 \mathrm{I}) n(\%)\end{array}$ \\
\hline 0 & $8(15.1)$ & $7(13.2)$ \\
$\mathrm{I}$ & $16(30.2)$ & $26(49.1)$ \\
2 & $16(30.2)$ & $15(28.3)$ \\
3 & $5(9.4)$ & $3(5.7)$ \\
4 & $8(15.1)$ & $2(3.8)$ \\
Mean score & 1.79 & 1.38 \\
\hline $\begin{array}{l}\text { Wilcoxon signed rank test (two-tailed): } Z=-2.82 \\
(P<0.005) .\end{array}$ &
\end{tabular}

\section{Overall ratings}

Table 3 compares overall ratings for psychiatric disorder between 1975/76 and 2001. Results indicate that the number of individuals receiving an overall score of 3 or 4 in 2001 had decreased from 1975/76, whereas the numbers who received a less severe score of 1 had increased. The Wilcoxon signed rank test found these differences to be significant.

Overall ratings from the 2001 data were also compared for frequency, management difficulty and severity scores on the CCB. Two-tailed Spearman's correlation coefficient $\rho$ indicated a significant correlation between overall ratings and frequency ratings $(\rho=0.331, P<0.05)$. No significant correlation was found for overall ratings and either management difficulty or severity ratings.

\section{Additional comparisons}

We also looked at the 2001 overall ratings in relation to the participants' age, gender, place of residence and level of intellectual disability. These figures are shown in Table 4. Comparison of scores by place of residence and age group both show a significant difference, with those living in the hospital and those aged 59 years and under, recording a higher mean overall rating for psychiatric disorder (for place of residence, those living in specialist provision were placed within the hospital group). No significant differences were found between males and females or levels of intellectual disability.

Finally, we compared the $1975 / 76$ data of those still living with those of the original cohort who are now deceased, in order to examine differences, if any, between these two groups and identify possible mortality trends. As an original cohort of 98, the living and deceased groups were fairly evenly matched. We found no significant differences between groups for overall ratings for psychiatric disorder. Among the deceased group, we did find a significantly lower level of psychiatrist ratings of stereotypy, eye avoidance, elation and overactivity, and a higher level of hostile irritability, but carer ratings showed no differences between the two groups. Our confidence in these differences is therefore limited.

An easier explanation would be that more individuals within the deceased group were of profound intellectual disability, and more were older at the baseline assessment period, which would account quite readily for any differential mortality.

\section{DISCUSSION}

The persistence of symptoms and psychiatric disorder

In this study, we were able to show that a high number of behavioural symptoms persisted over a 26-year period, particularly overactivity, which was noted by both 
Table 4 Comparison of cohort characteristics and overall psychiatric rating

\begin{tabular}{|c|c|c|}
\hline Variable & $\begin{array}{l}\text { Overall rating for psychiatric disorder } \\
\qquad \text { at } 200 \mathrm{I} n \text { (mean) }\end{array}$ & $\begin{array}{c}\text { Mann-Whitney } U \text { test } \\
\text { (two-tailed) }\end{array}$ \\
\hline Place of residence & & $Z=-2.77(P<0.0 \mathrm{I})$ \\
\hline Community & $42(1.17)$ & \\
\hline Hospital & II (2.18) & \\
\hline Age (years) & & $Z=-2.20(P<0.05)$ \\
\hline$\leqslant 59$ & $35(1.57)$ & \\
\hline$\geqslant 60$ & $18(1.11)$ & \\
\hline Gender & & NS \\
\hline Male & $21(1.48)$ & \\
\hline Female & $32(1.38)$ & \\
\hline Level of intellectual disability & & NS \\
\hline Severe & $26(1.08)$ & \\
\hline Profound & $18(1.44)$ & \\
\hline Not conclusive & $9(2.11)$ & \\
\hline
\end{tabular}

psychiatrists and carers to be significantly persistent. This is very much in accordance with clinical experience.

The cohort is clearly showing a similar behaviour pattern at both assessment points on psychiatrist ratings. From 1975/ 76 to 2001 three symptoms - slow, lability of mood and depressed - show notable increases. The other symptoms are remarkably persistent.

The carer ratings are less robust and can be somewhat misleading at first glance. Only three behaviour ratings were found to be persistent over time and the correlations were fairly weak. Figures from Table 1 (percentages of population showing each behaviour) show some behaviours where, at first glance, a perfect correlation would be expected, for example sleep disturbance. However, although the same number of individuals are showing that behaviour, they are not the same individuals. Careful consideration should therefore be given to dichotomous data such as this, because an exploration of numbers and/or percentages alone may lead to false demonstration of persistence.

It is interesting to note that overall ratings continue to show a high number of behavioural symptoms for the cohort, but the severity of these symptoms has decreased, suggesting that the group has become easier to manage. The high percentage of the cohort that has been relocated to the community supports this suggestion.

\section{Implications for care}

Any conclusions drawn from this study about the persistence of symptoms are obviously coloured by the use of older methodologies and definitions in 1975/76. Ways of investigating and measuring behavioural and psychiatric symptoms have improved in the intervening period. The comparison of overall ratings with the $\mathrm{CCB}$, however, does provide a measure of validity between overall ratings and frequency, and this allows us to be more confident in our findings.

It is predictable that individuals with higher levels of challenging behaviour are to be found within the hospital rather than community settings. In this study, individuals resident in hospital show, on average, almost twice as much challenging behaviour as individuals resident in the community. It is also predictable that decrease in severity with age. We found this to be the case, with individuals over 60 years of age being given lower overall ratings for psychiatric disorder. This is not surprising, given the noted increase in mobility problems from $1975 / 76$ to 2001 for the cohort as a whole.

\section{The passage of time}

In 1995, the second author concluded by stating that the passage of time was unlikely per se to bring about more than a modest abatement in the disordered behavioural symptoms are likely to behaviour of such a challenging group. This view is supported in this study by the continued persistence of the majority of the symptoms investigated. However, although the passage of time may not have fundamentally changed the behaviour patterns of this population with severe and profound intellectual disability, there has been a reduction in the severity of the symptomatology.

\section{APPENDIX}

Modified Manifest Abnormality Scale (MMAS; Ballinger et al, 1975)

The following list gives the 19 rating items (in bold) along with their definitions:

Slow, lacking spontaneity and definition delayed verbal responses, reduced motor activity

Suspicious, defensive reluctant to respond/ refusal/requests to leave interview situation

Histrionic exaggeration of symptoms for effect/ extravagant gestures/grossly manipulative behaviour

Depressed gloominess/clinically depressed/suicidal

Anxious/agitated apprehensive/in need of reassurance/inability to relax/motor agitation

Elated/euphoric cheerful, high spirits/marked elation of mood/infectious laughter and jollity

Flattened, incongruous lack of emotional tone/ impaired or incongruous emotional responses

Delusions/misinterpretations/thought disorder eccentric beliefs/over-valued ideas/ undoubted delusions/complex delusional symptoms

Hallucinations hypnogogic or eidetic images/ false perceptions with or without insight

Excessive concern with bodily functions over-concerned with health or bodily functions/ reluctance to move to other topics

Depressive thoughts morbid pessimism/feelings of inferiority, shame and guilt/nihilistic delusions

Overactivity fidgety, restless, pacing, frequent unnecessary movements

Distractibility stops talking, changes subject/ distracted by trivial noises or events outside of room/turns attention to furniture

Stereotypies constant repetition of movements or postures such as rocking, rubbing, nodding or grimacing

Hostile irritability uncooperative, irritable, angry, overtly hostile, discontented, haughty, antagonistic

Lability of mood whether lability of one mood or changing from one mood to another

Pica eating or attempting to eat substances that are not food

Self-injury activities that cause or are likely to cause actual bodily harm (e.g. hitting self, biting self, excessive scratching, pulling hair out, striking 
body on furniture, etc.)/excluding accidents and nailbiting

Eye avoidance failure to maintain eye contact during interview or failure to establish eye contact at all

\section{REFERENCES}

Ballinger, B. R., Armstrong, J., Presly, A. S., et a (1975) Use of a standardized psychiatric interview in mentally handicapped patients. British Journal of Psychiatry, 127, 540-544.

_\& Reid, A. H. (1977) Psychiatric disorder in an adult training centre and a hospital for the mentally handicapped. Psychological Medicine, 7, 525-528.

Day, K. A. \& Jancar, J. (1994) Mental and physical health and ageing in mental handicap: a review. Journal of Intellectual Disability Research, 38, 24I-256.

Goldberg, D. P., Cooper, B., Eastwood, M. R., et al (1970) A standardised psychiatric interview for use in community surveys. British Journal of Social \& Preventative Medicine, 24, 18-23.

Gunn, J. \& Fenton, G.W. (1969) Epilepsy in prison: a diagnostic survey. BMJ, iv, 326-328.

Harris, P., Humphreys, J. \& Thomson, G. (1994) A checklist of challenging behaviour: the developments of a survey instrument. Mental Handicap Research, 7(2), 118-133.

Holland A. J. (2000) Ageing and learning disability. British Journal of Psychiatry, I76, 26-31.

Jenkins, R., Rose, J. \& Jones, T. (1998) The checklist of challenging behaviour and its relationship with the psychopathology inventory for mentally retarded adults. Journal of Intellectual Disability Research, 42, 273-278.

Kiernan, C. \& Alborz, A. (1996) Persistence and change in challenging and problem behaviours of young adults with intellectual disability living in the family home. Journal of Applied Research in Intellectual Disabilities, $\mathbf{9}$ $181-193$

Moss, S., Emerson, E., Kiernan, C., et al (2000) Psychiatric symptoms in adults with learning disability and challenging behaviour. British Journal of Psychiatry I77, 452-456.

\section{CLINICAL IMPLICATIONS}

- Challenging behaviour is persistent over time, although its severity diminishes.

- The study serves as a baseline against which to assess the impact of treatment procedures.

- Severe challenging behaviour is an impediment to successful relocation to the community.

\section{LIMITATIONS}

- The methodology inevitably draws on an out-of-date protocol.

- The study utilised a trained, but different observer on this occasion, although this lent a degree of objectivity and reduced any practice effect.

- Carer ratings were less convincing than observer ratings.

CHRISTINA L. THOMPSON, Dip Psych, Tayside Primary Care NHS Trust, Clinical Psychology Department, Wedderburn House, Dundee; ANDREW REID, FRCPsych, Carseview Centre, Ninewells Medipark, Dundee

Correspondence: Dr Andrew Reid, Carseview Centre, 4 Tom McDonald Avenue, Ninewells Medipark, Dundee DD2 INH, UK

(First received II October 200I, final revision 12 March 2002, accepted 19 March 2002)

Nottestad, J. A., Stromgren, B. \& Linaker, O. M. (2000) Psychiatric and behavioral disturbances in elderly mentally retarded before and after deinstitutionalization. Nord Journal of Psychiatry, 54, 28I-286.

Reid, A. H., Ballinger, B. R. \& Heather, B. B. (1978) Behavioural syndromes identified by cluster analysis in sample of 100 severely and profoundly retarded adults. Psychological Medicine, 8, 399-412.

, _ , et al (1984) The natural history of

behavioural symptoms among severely and profoundly mentally retarded patients. British Journal of Psychiatry, 145, 289-293.

_ \& _ (1995) Behaviour symptoms among mentally retarded patients. A 16-18 year follow-up study. British Journal of Psychiatry, 167, 452-455.

Rutter, M. \& Graham, P. (1968) The reliability and validity of the psychiatric assessment of the child. I. Interview with the child. British Journal of Psychiatry, II4 563-579. 